\title{
Variational cartographic projections for the Slovak Republic territory
}

\author{
Margita Vajsáblová and Daniel Szatmári \\ Department of Mathematics and Descriptive Geometry, Faculty of Civil Engineering, Slovak University of Technology, \\ Bratislava, Slovak Republic.
}

Received 3 January, 2014; Accepted 5 March, 2014

\begin{abstract}
Disadvantages of currently used Křovák`s projection in Slovak Republic such as large scale distortion became evident after dividing of Czechoslovakia. The new proposal of the cartographic projection for the Slovak Republic (Lambert's conformal conic projection in normal position) was created in 2010. The aim of this paper is to define an optimal oblique position of map projections for the Slovak Republic territory and to design two cartographic projections in different positions (normal, oblique) using variational criteria modified for conformal projections for the Slovak Republic. Used calculus, the achieved scale distortions and their comparison with scale distortions of currently used map projections will be demonstrated.
\end{abstract}

Key words: Conformal cartographic projection, variational criteria, scale distortion, variational cartographic projection.

\section{INTRODUCTION}

The currently used cartographic projection in the Slovak Republic (and also in the Czech Republic) is the Křovák`s projection which is an oblique case of a conformal conic projection based on two preserved parallels and was designed in 1922 by Josef Krrovák strictly for Czechoslovakia. The Bessel's reference ellipsoid is transformed to a sphere using Gauss' conformal projection. Then, the sphere is transformated to a secant cone in oblique position. The coordinates are positive to the south and west (Srnka, 1986). Disadvantages of this projection such as large scale distortion became evident after dividing of Czechoslovakia. In 2010 a new projection, Lambert's projection with parameters for Slovakia was designed at the request of the Geodesy, Cartography and Cadastre Authority of the Slovak Republic by Margita Vajsáblová. This new conformal projection uses a normal cone, secant to the reference ellipsoid GRS 1980 and was designed for the Slovak Republic territory (Vajsáblová, 2011). The reference ellipsoid is transformed directly on a secant cone in normal position. The coordinates are positive to the north and east.

One of the basic problems in cartography is the problem of optimal map projections, especially in the area 
of the creation of large-scale maps and partially mediumscale maps, that is, those projections of a given domain on a reference surface onto the plane under which the distortion is minimal. The most important factors of selection of map projection are shape, size and position of projected territory, also purpose and scaling factor of the map. The aim of this paper is to design two map projections in different positions (normal, oblique) using variational criteria modified for conformal projections for the Slovak Republic. Examples of using these criteria to derive and optimize map projections for other territories (Canada, Czech Republic) and their valuation and comparison are described in Frankich (1982), Bořík (1999) and Hojovec et al. (1975).

The optimal oblique position of cartographic projection for the Slovak Republic territory is defined in this paper and the aim is to design two cartographic projections in different positions (normal, oblique) using variational criteria modified for conformal projections for the Slovak Republic. Used calculus, the achieved scale distortions and their comparison with scale distortions of currently used map projections will be demonstrated.

\section{Valuation of cartographic projections}

The type of criteria for deciding the merits of map projections are as follow (Hojovec et al., 1987):

a) Extremal and minimaximal,

b) Variational (additive, integral) criteria.

In extremal criteria we evaluate conformal projection by:

i. Maximal value of scale distortion: $|m-1|_{\max }$,

ii. Maximal value of area distortion: $\left.\left|m_{p l}-1\right|\right|_{\max }$.

The equivalent projections are evaluated by maximal value of angle distortion: $|\Delta \omega|_{\max }$.

Chebyshev's theorem for conformal projection, in which natural logarithm of scale distortion is the least different from zero, if scale distortion of closed boundary curve is constant (Chebyshev, 1962). These projections are called minimaximal type projections.

Meshcheryakov's theorem for Euler's type projections (orthogonal equivalent): the best of Euler's projection by minimaximal criterion is the projection in which image of the central meridian is a line and the scale distortion in every points holds, that scale distortion of meridian and parallel are equal to 1 (Meshcheryakov, 1968).

The most popularized variational type criteria for valuation of map projections are the following:

i. The Airy`s criterion (1861), where $h^{2}$ is mean quadratic scale distortion defined by extremal scale factors $m_{a}, m_{b}$ : $h^{2}=\frac{1}{2}\left[\left(m_{a}-1\right)^{2}+\left(m_{b}-1\right)^{2}\right]$,

ii. The Jordan's criterion, which characterizing scale distortion in a point by $h^{2}$ for all the azimuths $A$ :

$h^{2}=\frac{1}{2 \pi} \int_{0}^{2 \pi}\left(m_{A}-1\right)^{2} \mathrm{~d} A$,

in some case for several value of the azimuth $A$ :

$h^{2}=\frac{1}{\mu} \sum\left(m_{A}-1\right)^{2}$,

where $m_{A}$ is factor of scale distortion appertaining to direction with azimuth $A, \mu$ is number of these directions. iii. The Kavraiskii's criteria are modification of previous criteria by using the logarithmic values (Kavraiskii, 1959):

- the Airy-Kavraiskii criterion:

$h^{2}=\frac{1}{2}\left[\ln ^{2} m_{a}+\ln ^{2} m_{b}\right]$,

- the Jordan-Kavraiskii criterion:

$h^{2}=\frac{1}{2 \pi} \int_{0}^{2 \pi} \ln ^{2} m_{A} \mathrm{~d} A$,

$h^{2}=\frac{1}{\mu} \sum \ln ^{2} m_{A}$

Characteristic value of cartographic projection of domain $\Delta$ of reference surfaces is value of integral $l$ :

$I=\frac{1}{\Delta} \iint_{\Delta} h^{2} \cos U \mathrm{~d} U \mathrm{~d} V$

or for $n$ choices points:

$I=\frac{1}{n} \sum h^{2}$

Mentioned criteria are possible to use in deriving of cartographic projection too, thereby we obtain variational type of projection (Urmaev, 1947).

The conformal projections are frequently applied in coordinate systems, because in this type of projection holds, that scale distortion is independent to azimuth, we 
are able to simplify previous formulae in the next chapters.

\section{Variational cartographic projections}

We are concerned with creation of conformal map projections of territory with minimal scale distortion by variational Criterion (7), where we apply the AiryKavraiskii Criterion (4) modified for conformal projection $\left(m_{a}=m_{b}=m\right)$ :

$h^{2}=\ln ^{2} m$.

Projected territory is situated on reference sphere between parallels with geographic latitude $U_{S}, U_{N}$ and between meridians with geographic longitude $V_{W}$ and $V_{E}$, where we use their relative value from central meridian $V_{0}$ :

$V_{0}=\frac{V_{W}+V_{E}}{2}$.

In consideration of symmetry of spherical trapezium and after substitution of (9) to variational Criterion (7) we obtain:

$I=\frac{1}{\Delta} \int_{0}^{V_{1}} \int_{U_{S}}^{U_{N}} \ln ^{2} m \cos U \mathrm{~d} U \mathrm{~d} V$,

where $V_{1}$ is maximum of relative geographic longitude. We need to find function In $m$ from condition that value of $I$ is minimal.

In conformal projection it holds (Hojovec et al. 1975):

$\frac{\partial^{2} u}{\partial Q^{2}}+\frac{\partial^{2} u}{\partial V^{2}}=0$

where:

$u=\ln m \cos U$

and $Q$ is isometric latitude calculated by:

$Q=\ln \operatorname{tg}\left(\frac{U}{2}+45^{\circ}\right)$

Solution of Equation (12) has a shape (Hojovec et al., 1975): $u=\sum_{j=0}^{n}\left(a_{j} \psi_{j}+b_{j} \tau_{j}\right)$

where $\psi_{j}$ and $T_{j}$ were determine by:

$\psi_{j}+i \tau_{j}=(Q+i V)^{j}$.

Two complex variables are equal than its imaginary and real components are equal, for $\psi_{j}$ and $\tau_{j}$ we obtain $(j=0$ to $j=4$ ):

$\begin{array}{llll}\psi_{0}=1, & \psi_{1}=Q, & \psi_{2}=Q^{2}-V^{2}, & \psi_{3}=Q^{3}-3 Q V^{2}, \\ \psi_{4}=Q^{4}-6 Q^{2} V^{2}+V^{4} ; & \tau_{0}=0, & \tau_{1}=V, & \tau_{2}=2 Q V,\end{array}$

$\tau_{3}=3 Q^{2} V-V^{3}, \quad \tau_{4}=4 Q^{3} V-4 Q V^{3}$.

Equation (15) is simplified for symmetric:

$u=\sum_{j=0}^{n} a_{j} \psi_{j}$

After substitution Equations (13) and (18) to AiryKavraiskii criterion (11) we have:

$I=\frac{1}{\Delta} \int_{0}^{V_{1} U_{N}} \int_{U_{S}}\left(\sum_{j=0}^{4} a_{j} \psi_{j}-\ln \cos U\right)^{2} \cos U \mathrm{~d} U \mathrm{~d} V$

By minimalisation of (19) we obtain system of five equations for $j=0$ to 4 , where $j$ is:

$a_{0} P_{j 0}+a_{1} P_{j 1}+a_{2} P_{j 2}+a_{3} P_{j 3}+a_{4} P_{j 4}=F_{j}$,

where functions $P$ and $F$ hold (Hojovec et al. 1975):

$P_{j k}=\int_{0}^{V_{1}} \int_{U_{S}}^{U_{N}} \psi_{j} \psi_{k} \cos U \mathrm{~d} U \mathrm{~d} V, \quad F_{k}=\int_{0}^{V_{1}} \int_{U_{S}}^{U_{N}} \psi_{k} \cos U \ln \cos U \mathrm{~d} U \mathrm{~d} V$.

The coefficients $a_{j}$ are calculated for domain defined by marginal parallels $U_{S}, U_{N}$ and meridians $0^{\circ}, V_{1}$. The factor of scale distortion is calculated by:

$\ln m=\sum_{j=0}^{4} a_{j} \psi_{j}-\ln \cos U$.

Proposal of two variational cartographic projections for the Slovak Republic

The geographic coordinates $\varphi, \lambda$ of the boundary points of the Slovak Republic on reference ellipsoid GRS 1980, 


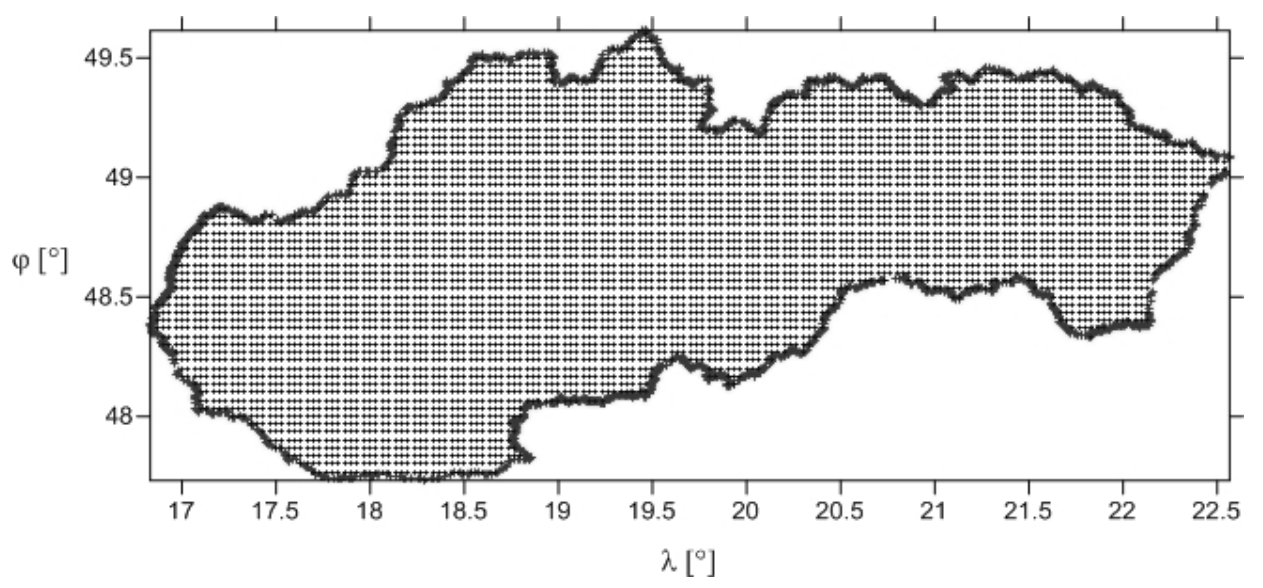

Figure 1. Test points for calculating the factor of scale distortion.

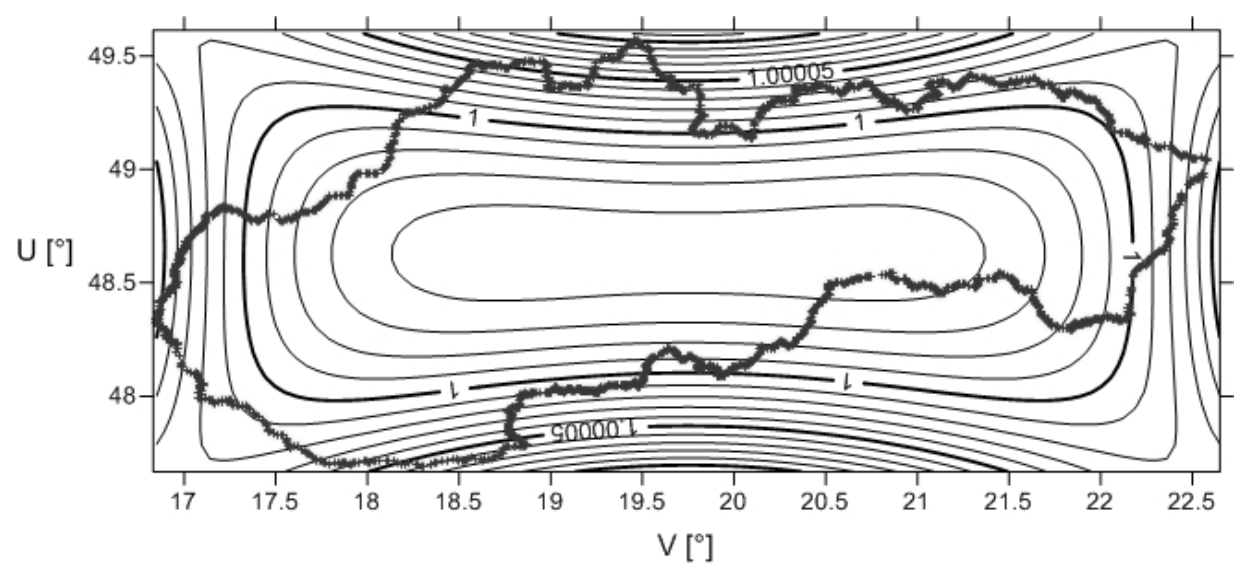

Figure 2. Curves of constant scale distortion - normal case of variational cartographic projection.

we have transformated on the spherical coordinates $U, V$ by Gauss` conformal projection:

$\operatorname{tg}\left(\frac{U}{2}+45^{\circ}\right)=k\left[\operatorname{tg}\left(\frac{U}{2}+45^{\circ}\right) \sqrt{\left(\frac{1-e \sin \varphi}{1+e \sin \varphi}\right)^{e}}\right]^{\alpha}, \quad V=\alpha \cdot \lambda$

with parameters: $\alpha=1.0006405967505$ and $k=1.003$ 3159956373.

Preserved parallel has geographic latitude $\varphi_{0}=48^{\circ} 40^{\prime} 21.2520^{\prime \prime}$ and radius of the sphere is $R=6380840.7213 \mathrm{~m}$.

The Slovak Republic is situated on this sphere between parallels with geographic latitude $U_{S}=47^{\circ} 41^{\prime} 27.0720^{\prime \prime}$, $U_{N}=49^{\circ} 34^{\prime} 15.7881^{\prime \prime}$ and between meridians with geographic longitude $V_{W}=16^{\circ} 50^{\prime} 32.3600^{\prime \prime}$ and $V_{E}$ $=22^{\circ} 34^{\prime} 50.6852^{\prime \prime}$, where we have used their relative values from central meridian $V_{0}$ calculated as the arithmetic mean (10).

Values of evaluated coefficients $a_{j}$ for the first domain defined by marginal parallels $U_{S}, U_{N}$ and meridians $0^{\circ}, V_{1}$ and with using Equations (11) to (21) are:

$a_{0}=+17.334734, \quad a_{1}=-70.610239, \quad a_{2}=+107.550822$,

$a_{3}=-73.595801, \quad a_{4}=+18.886817$.

All of the calculus has been realized by system Wolfram Mathematica 8 . We have calculated the factor of scale distortion for 5424 points (Figure 1) by Equation (22), the curves of constant scale distortion have been visualized in Surfer 9 (Figure 2).

The second variational projection is proposed in oblique position, therefore on domain defined on reference sphere between two cartographic parallels with 


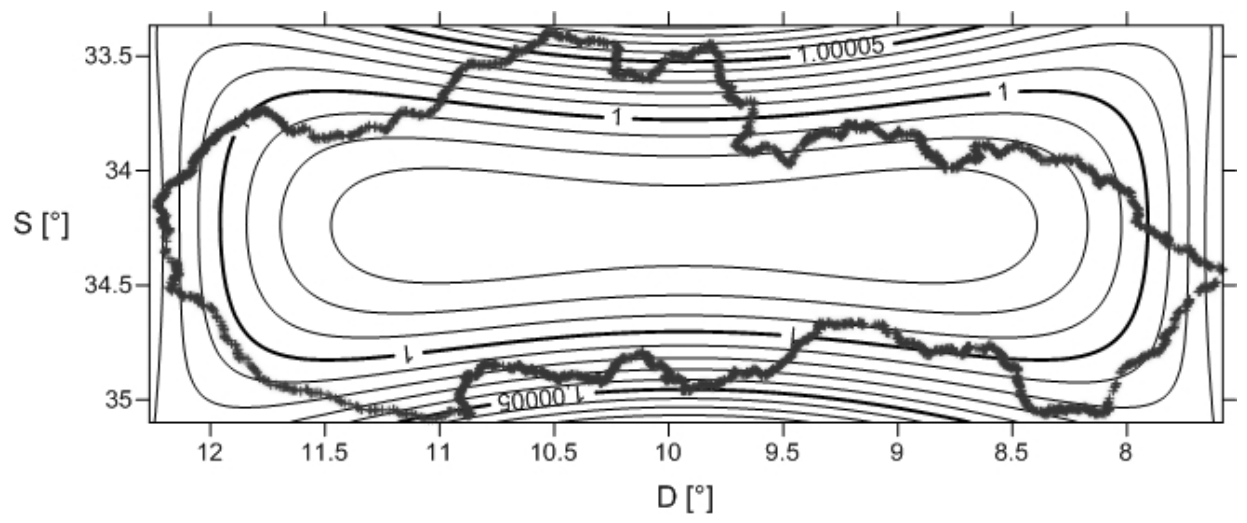

Figure 3. Curves of constant scale distortion - oblique case of variational cartographic projection.

cartographic latitude $S_{S}, S_{N}$ and two cartographic meridians with cartographic longitude $D_{W}, D_{E}$. We have defined the values of this cartographic coordinates by consecutive steps:

$1^{\text {st }}$ We have chosen three points $A, B, C$ on the south boundary of the Slovak Republic with geographic coordinates:

$$
\begin{aligned}
& A\left[U_{1}=48^{\circ} 19^{\prime} 49.9601^{\prime \prime}, V_{1}=22^{\circ} 05^{\prime} 58.6244^{\prime \prime}\right], \\
& B\left[U_{2}=48^{\circ} 17^{\prime} 28.5876^{\prime \prime}, V_{2}=21^{\circ} 49^{\prime} 51.4052^{\prime \prime}\right], \\
& C\left[U_{3}=47^{\circ} 42^{\prime} 47.2572^{\prime \prime}, V_{3}=18^{\circ} 30^{\prime} 50.1205^{\prime \prime}\right]
\end{aligned}
$$

$2^{\text {nd }}$ We have approximated the cartographic parallel circle through the points $A, B, C$ and have calculated the geographic coordinates $U_{K}$ and $V_{K}$ of cartographic pole $K$ by equation:

$$
\begin{gathered}
\operatorname{tg} V_{k}=\frac{\left(\cos U_{1} \cos V_{1}-\cos U_{3} \cos V_{3}\right)\left(\sin U_{1}-\sin U_{2}\right)-}{\left(\cos U_{1} \sin V_{1}-\cos U_{2} \sin V_{2}\right)\left(\sin U_{1}-\sin U_{3}\right)-} \ldots \\
\ldots \frac{-\left(\cos U_{1} \cos V_{1}-\cos U_{2} \cos V_{2}\right)\left(\sin U_{1}-\sin U_{3}\right)}{-\left(\cos U_{1} \sin V_{1}-\cos U_{3} \sin V_{3}\right)\left(\sin U_{1}-\sin U_{2}\right)}, \\
\operatorname{tg} U_{k}=\frac{\cos U_{2} \cos \left(V_{k}-V_{2}\right)-\cos U_{1} \cos \left(V_{k}-V_{1}\right)}{\sin U_{1}-\sin U_{2}} .
\end{gathered}
$$

from that $U_{K}=-5^{\circ} 53^{\prime} 41.1964^{\prime \prime}$ and $V_{K}=$ $32^{\circ} 08^{\prime} 18.5219^{\prime \prime}$.

$3^{\text {rd }}$ Then we have transformated geographic coordinates $U, V$ of the points of Slovakia boundary on cartographic coordinates $S, D$. Extremal values of cartographic latitudes $S_{S}, S_{N}$ and cartographic longitudes $D_{W}, D_{E}$ are the coordinates of marginal parallels and marginal meridians of boundary trapezium. The Slovak Republic is situated on the sphere between parallels with cartographic latitude $S_{S}=35^{\circ} 05^{\prime} 05.7509^{\prime \prime}, \quad S_{N}$ $=33^{\circ} 23^{\prime} 42.1307^{\prime \prime}$ and between meridians with cartographic longitude $D_{W}=12^{\circ} 13^{\prime} 59.6088^{\prime \prime}, D_{E}=7^{\circ} 34^{\prime}$ $55.6498^{\prime \prime}$, where we have used their relative values from central meridian $D_{0}$ calculated as the arithmetic mean:

$D_{0}=\frac{D_{W}+D_{E}}{2}$,

from where the maximal value of relative cartographic longitude is $D_{1}$. The interval of latitudes is $1^{\circ} 52^{\prime} 48.7161^{\prime \prime}$ in normal position, $1^{\circ} 41^{\prime} 23.6202^{\prime \prime}$ in oblique position.

The coefficients $a_{j}$ have been evaluated for the second domain defined by marginal parallels $S_{S}, S_{N}$ and meridians $0^{\circ}, D_{1}$ by Equations (11) to (21) where the following substitutions have been used:

$U=S, \quad V=D, \quad U_{S}=S_{S}, \quad U_{N}=S_{N}, \quad V_{1}=D_{1}$.

Results of coefficients $a_{j}$ :

$a_{0}=+6.800198, \quad a_{1}=-42.196398, \quad a_{2}=+98.029304$,

$a_{3}=-102.608260, a_{4}=+40.284538$.

All the calculus have been realized by system Wolfram Mathematica 8 . We have calculated the factor of scale distortion for 5424 points (Figure 1) by Equation (22) where $U=S$, the curves of constant scale distortion have been visualized in Surfer 9 (Figure 3).

We have compared these variational cartographic projections with the currently used projection defined by the law - Křovák's projection (Srnka, 1986). The Křovák's projection is an oblique case of conformal conic projection based on two preserved parallels designed in 
Table 1. Extremal scale distortions in cartographic projections.

\begin{tabular}{ll}
\hline Cartographic projection & Scale distortion $[\mathbf{c m} / \mathbf{k m}]$ \\
\hline Variational - normal & From -4.6 to +9.7 \\
Variational - oblique & From -3.8 to +7.2 \\
Křovák & From -10.0 to +11.0 \\
Lambert & From -6.7 to +6.7 \\
\hline
\end{tabular}

Table 2. Scale distortions and their quadratic values in cartographic projections by additive criterion.

\begin{tabular}{lcc}
\hline \multirow{2}{*}{ Cartographic projection } & \multicolumn{2}{c}{ Additive criterion } \\
\cline { 2 - 3 } & $\mathbf{c m}^{\mathbf{2}} / \mathbf{k m}^{\mathbf{2}}$ & $\sqrt{I} \mathbf{[ \mathbf { c m } / \mathbf { k m } ]}$ \\
\hline Variational - normal & 8.667 & 2.944 \\
Variational - oblique & 6.076 & 2.465 \\
Křovák & 51.125 & 7.150 \\
Lambert & 25.489 & 5.049 \\
\hline
\end{tabular}

Table 3. Scale distortions and their quadratic values in cartographic projections by integral criterion.

\begin{tabular}{lcc}
\hline \multirow{2}{*}{ Cartographic projection } & \multicolumn{2}{c}{ Integral criterion } \\
\cline { 2 - 3 } & $\mathbf{c m}^{2} / \mathbf{k m}^{2}$ & $\sqrt{I}[\mathbf{c m} / \mathbf{k m}]$ \\
\hline Variational - normal & 8.709 & 2.951 \\
Variational - oblique & 6.131 & 2.476 \\
Křovák & 48.375 & 6.955 \\
Lambert & 26.211 & 5.120 \\
\hline
\end{tabular}

1922 by Josef Křovák. Used in the Czech Republic and Slovakia. In 2010 a new projection, Lambert's projection with parameters for Slovakia (Vajsáblová, 2011) was designed at the request of the Geodesy, Cartography and Cadastre Authority of the Slovak Republic. This new conformal projection uses a normal cone, secant to the reference ellipsoid and was designed for the Slovak Republic territory by Margita Vajsáblová. The results are demonstrated in Tables 1 to 3 . The valuation of scale distortions and their quadratic values in the metioned cartographic projections by additive criterion has been calculated by Equation (8) for $n=5424$ points (Table 2) and by integral criterion by Equation (7), where region $\Delta$ is limited by boundaries of the Slovak Republic (Table 3).

\section{Conclusions}

Disadvantages of currently used Křovák`s projection such as large scale distortion became evident after dissolution of Czechoslovakia. The maximal scale distortion of this projection is about $4.3 \mathrm{~cm} / \mathrm{km}$ larger than scale distortion of Lambert's projection with parameters for the Slovak Republic also about $3.8 \mathrm{~cm} / \mathrm{km}$ larger than scale distortion of oblique variational projection. Valuation of these map projections by variational criteria (additive or integral) demonstrated in the Tables 2 and 3 also confirms their suitability.

The most suitable cartographic projection of territory of the Slovak Republic by extremal criteria is the Lambert's projection with parameters for the Slovak Republic. The most suitable map projection by variational criteria is the above described variational projection in oblique position.

\section{Conflict of Interests}

The author(s) have not declared any conflict of interests.

\section{ACKNOWLEGEMENT}

This article is one of the outputs of the $1 / 1063 / 11$ project 


\section{supported by the VEGA Grant Agency.}

\section{REFERENCES}

Airy GB (1861). Explanation of a projection by balance of errors for maps applying to a very large extent of the Earth's surface and comparison with other projections. Philosophical Magaz. J. Sci. 22:409-421.

Bořík M (1999). Using of variational criteria for projection of CR. Map product of geoinformatic technologies: Proceedings of the 13th Cartographic Conference. Bratislava. pp. 37-42.

Chebyshev PL (1962). Sur la construction des cartes géographiques. Oeuvres de P.L. Chebyshev. Chelsea.

Frankich K (1982). Optimization of geographic map projections for Canadian territory, Simon Fraser University, Canada.

Hojovec V, Klášterka J, Rendlová H (1975). Using of Chebyshev's variational criterion in conformal projection. Geodetic cartographic Rev. 21(63):3-6.
Hojovec V, Daniš M, Hájek M, Veverka B (1987). Cartography. Praha (Geodetic and Cartographic Enterprise).

Kavraiskii VV (1959). Collected works 2. Moscow.

Meshcheryakov GA (1968). Theoretical foundations of mathematical cartography. Moscow.

Srnka E (1986). Mathematical cartography. Military Academy of Antonín Zápotocký, Brno.

Urmaev NA (1947). Methods for finding new cartographic projections. Moscow.

Vajsáblová M (2011). Proposal of new cartographic projection of Slovak Republic. Geodetic and cartographic Rev. 57(99):185-190. 